\section{Nachhaltige Investitionen mit marktgerechter Rendite?}

Investitionen im Nachhaltigkeitsbereich werden heute zumeist anhand umfangreicher Bewertungen mit mehr als 100 Kriterien analysiert. Doch die Konzentration auf die zentralen Treiber für die Finanzperformance könnte nachhaltigen Geldanlagen den Weg zum Mainstream öffnen. Von Axel Hesse

$\mathbf{N}$ achhaltige Investments sind zwar auf dem Vormarsch, der Weg aus der Nische zur Gesamtmarktdurchdringung, also in den Mainstream, wird jedoch nur dann gelingen, wenn langfristig eine marktgerechte Rendite oder besser eine risikoadjustierte Outperformance gegenüber der Benchmark erzielt wird. Denn für die große Mehrheit institutioneller Investoren sind und bleiben Rendite und Risiko die wichtigsten Parameter jeglichen Investments.

Nachhaltigkeitskriterien wie Umwelt-, Arbeitnehmer- und Entwicklungsbelange werden nur dann verstärkt in den Investmentprozess integriert werden, wenn sie für die finanzielle Performance der Investments von Bedeutung sind. Doch für welche Nachhaltigkeitskriterien gilt dies?

\section{Schlüsselindikatoren für die Finanzperformance}

Die gleiche Frage stellte sich auch aus Sicht der EU-Bilanzrichtlinien 78/660/ EWG und 83/349/EWG für große Kapitalgesellschaften. Denn diese müssen in ihrem Geschäfts- beziehungsweise Lagebericht einer Berichtspflicht für „nichtfinanzielle Leistungsindikatoren “ entsprechen, „die für die betreffende Geschäftstätigkeit von Bedeutung sind, einschließlich Informationen in Bezug auf Umwelt- und Arbeitnehmerbelange. "In der englischen Fassung der Richtlinien werden „Leistungsindikatoren“ treffender als „Key Performance Indicators“ (KPIs) bezeichnet. Diese Richtlinien wur- den in den EU-Mitgliedsstaaten umgesetzt, beispielsweise in Deutschland (ID 289; 315 HGB) und Österreich (\$』 243; 267 HGB).

Seit der Änderung im deutschen HGB Ende 2004 hat Sustainable Development Management, kurz SD-M®, in umfangreichen Untersuchungen die drei branchenspezifischen KPIs für eine nachhaltige Entwicklung bestimmt, welche für das Geschäftsergebnis und die voraussichtliche Entwicklung der Unternehmen in den nächsten fünf Jahren am bedeutendsten sind. Die Definition der SDKPIs erfolgte unter Beteiligung von Deloitte, Bundesumweltministerium und folgender Investment- und Researchhäuser, welche weltweit mehr als zwei Billionen Euro an nachhaltigen Investments beeinflussen: Crédit Agricole Cheuvreux, Dexia Asset Management, Ethix SRI Advisors, GES Investment Services, Hermes, imug/EIRIS, KLD Research \& Analytics, RiskMetrics Group, Sarasin, Social Investment Forum Japan, Société Générale, Sustainalytics und Vigeo.

\section{Investierbarer Standard für zahlreiche Branchen}

Die SD-KPIs stellen aus Sicht der Investoren, Analysten und des Bilanzrechts eine Mindestberichtsanforderung für Unternehmen im Lagebericht dar. So sind SD-KPIs mittlerweile das achtwichtigste Wettbewerbskriterium des jährlichen Wettbewerbs „Der beste Geschäftsbericht“ des Manager Magazins unter der wissenschaftlichen Leitung von Prof. Dr. Dr. h.c. Jörg Baetge.

Der 2010 veröffentlichte „SD-KPI Standard 2010-2014“ beinhaltet die drei wichtigsten SD-KPIs für die finanzielle Performance der 68 Industrien des Global Industry Classification Standard (GICS). GICS wurde von Morgan Stanley Capital International und Standard \& Poor's gemeinsam entwickelt und wird für rund die Hälfte der weltweiten Indexinvestments verwandt. Der urheberrechtlich geschützte „SD-KPI Standard 20102014“ stellt somit einen investierbaren,

Abbildung 1: Outperformance-Erwartung für fokussierte Ansätze nachhaltigen Investments

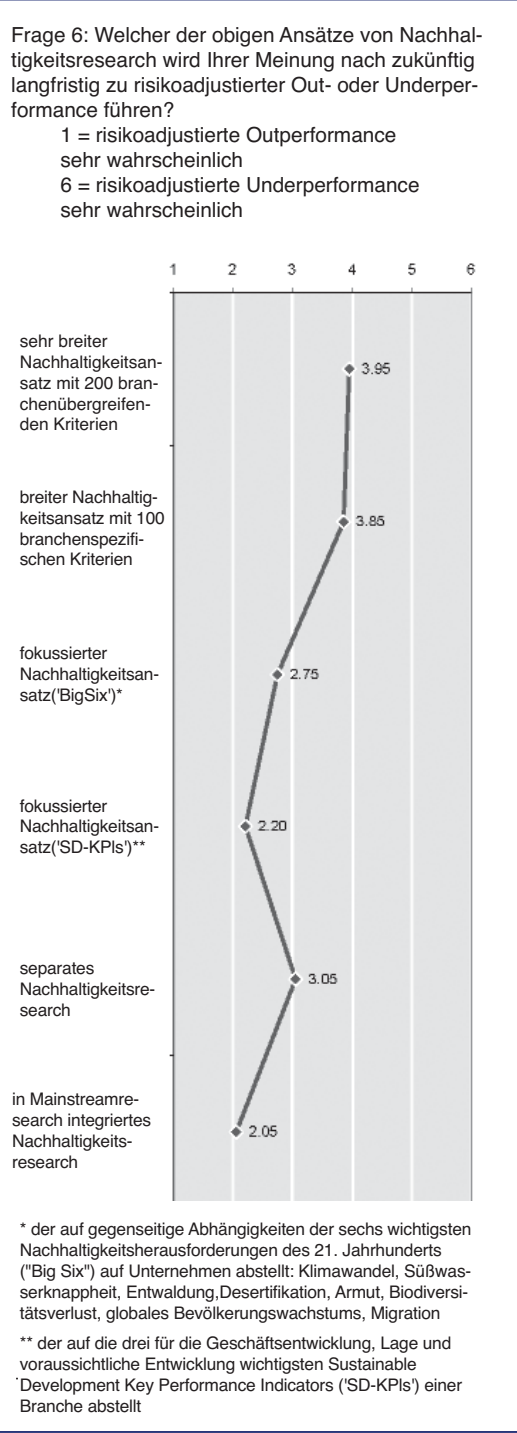
Branche abstelt

Quelle: Hesse 2008 
Abbildung 2: Beispiele für „Sustainable Development Key Performance Indicators (SD-KPIs) mit besonders hoher Bedeutung für die Finanzperformance

\begin{tabular}{lll}
\hline & Kurzbezeichnung & ökonomische Bedeutung \\
\hline $\begin{array}{ll}\text { SD.KPI 1 der } \\
\text { Automobilbranche }\end{array}$ & Flottenverbrauch & $\begin{array}{l}\text { Umsatzsteigerungen von Modellen mit gerin- } \\
\text { gen Verbrauchswerten bei höheren Ölpreisen }\end{array}$ \\
& & in 2008 sowie Abwrackprämien in 2009. \\
& & Umsatzeinbußen bei Modellen mit hohen Ver- \\
brauchswerten, z.B. bei US-amerikanischen & \\
& & oder Premium-Herstellern \\
\hline SD.KPI 1 der & konzernweite Treibhausgasinten- & Kosten für Kauf von Emissionsrechten, z.B. \\
Versorger & sität der Energieerzeugung & bei RWE in 2008 ca. 1Milliarde Euro \\
\hline SD·KPI 2 der & SD-Kreditrisiken- und Kreditchan- & Die Kontroversen bezüglich "Sub-Prime" oder \\
Banken & cenprüfung im Retail Banking & "räuberischen" Verleihpraktiken, welche den \\
& & Ausgangspunkt der gegenwärtigen Finanzkri- \\
& & se bildeten, wurden bereits im Oktober 2006 \\
& & für SO·KPI 2 definiert. \\
\hline
\end{tabular}

Quelle: Bundesumweltministerium 2009

über alle Branchen breit diversifizierten Best-in-Class-Standard für performanceorientiertes nachhaltiges Investment dar (1). Die bisher häufiger vorherrschende Unsicherheit bei der Definition nachhaltiger Investments wird transparent und fokussiert auf die primären Investmentziele Rendite und Risiko geklärt.

\section{Nachhaltiges Alpha für Mainstream-Investment}

Führende europäische Pensionsfondsmanager die 460 Milliarden an Assets managen bescheinigen den fokussierten nachhaltigen Investmentansätzen eine hohe Outperformance-Wirkung (siehe Abbildung 1). Die von SD-M® entwickelten Ansätze fokussieren branchenorientiert auf die SD-KPIs oder themenspezifisch auf die sechs wichtigsten Herausforderungen für nachhaltige Entwicklung im 21. Jahrhundert, die so genannten Big Six.

Im Gegensatz dazu stehen die heute noch überwiegend verwendeten, sehr breiten Nachhaltigkeitsresearch-Ansätze mit 100 oder 200 Kriterien. Hier erwarten die europäischen Pensionsfonds eine leichte Underperformance. Begründet wird dies mit hohen Kosten für das umfangreiche Research, zu hoher Komplexität und der Tatsache, dass die bedeutendsten Treiber für die nachhaltige Finanzperformance in der unumgänglichen Gewichtung der zu vielen Kriterien untergehen. Die Überfrachtung mit Kriterien wird so zur finanziellen Perfor- mancebremse. SD-KPI- oder Big Six-Ansätze werden hingegen als Quelle für nachhaltiges Alpha angesehen. Die drei überwiegend quantitativ definierten SDKPIs sind auch ideal für die Integration in Mainstream-Research geeignet. Denn die konventionellen Analysten müssen sich wie das Top-Management von Unternehmen auf Kernherausforderungen einer Branche konzentrieren.

\section{Sub-Prime-Risiken schon 2006 integriert}

Die drei Beispiele in Abbildung 2 verdeutlichen die Performancewirkung von SD-KPIs. Besonders markant ist sicherlich, dass bereits im Oktober 2006 SubPrime-Risiken in die SD-KPIs der Banken integriert wurden.

\section{Anmerkungen}

(1) Download des SD-KPI Standard 2010-2014 im Internet unter: http://www.SD-M.de

\section{Literatur}

Bundesumweltministerium (Hrsg.): Was Investoren wollen - Nachhaltigkeit in der Lageberichterstattung. Berlin 2009.

Hesse, A.: Langfristige und nachhaltige Altersvorsorgeinvestments - Eine Studie führender, europäischer Pensionsfonds. Zug 2008.

\section{AUTOR + KONTAKT}

Dr. Axel Hesse ist Senior Consultant bei SD-M Sustainable Development Management.

SD-M, Am Krug 30, 48151 Münster, Germany Tel.: +49 251 2394678, E-Mail: Hesse@SD-M.de, Internet: http://www.SD-M.de

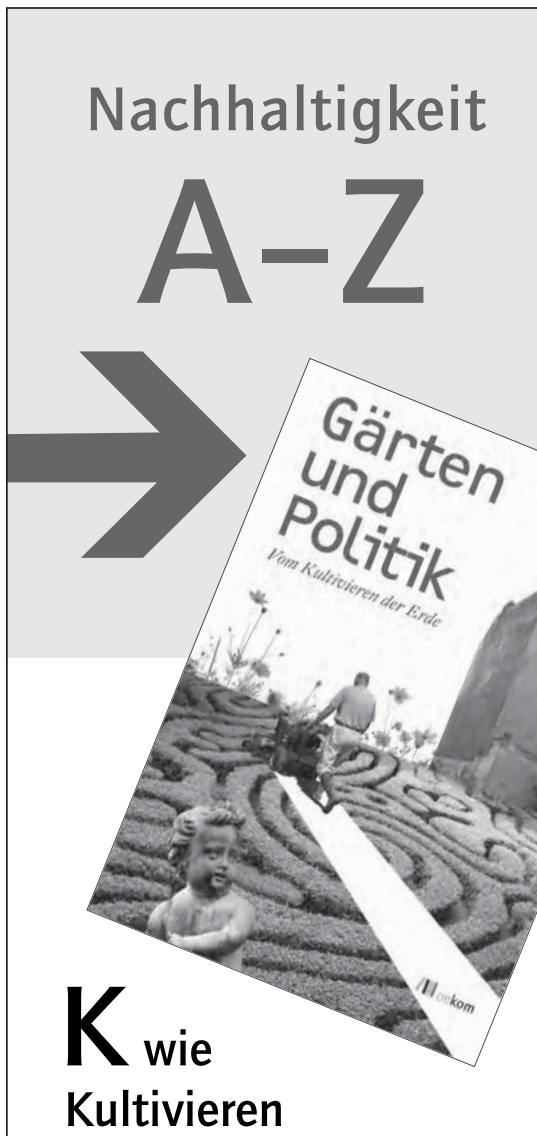

Gärten und Städte entstanden als Verteidigungsorte des Menschen gegen die wilde Natur. Mit deren Aneignung wandelten sich auch die menschliche Natur und das gesellschaftliche Leben.

Expert(inn)en verschiedener Fachrichtungen zeichnen in diesem Buch ein facettenreiches Bild vom Garten - von der Renaissance bis zur Gegenwart, vom humanistischen Garten bis heute: Bunt wie das Leben selbst und reich an Erkenntnissen über das, was uns bewegt.

B. Reimers (Hrsg.)

Gärten und Politik

Vom Kultivieren der Erde

320 Seiten, mit vielen farbigen

Abbildungen, 29,90 EUR,

ISBN 978-3-86581-158-5

Erhältlich bei

www.oekom.de

kontakt@oekom.de

\section{/ Illoekom}

Die guten Seiten der Zukunft 
(c) 20I0 Authors; licensee IÖW and oekom verlag. This is an article distributed under the terms of the Creative Commons Attribution Non-Commercial No Derivates License (http://creativecommons.org/licenses/by-nc-nd/3.o/), which permits unrestricted use, distribution, and reproduction in any medium, provided the original work is properly cited. 